\title{
Dynamique de bulles traversant l'interface séparant deux liquides
}

\author{
Romain Bonhomme ${ }^{1,2, a}$, Jacques Magnaudet ${ }^{2}$ et Bruno Piar ${ }^{1}$ \\ 1 Institut de Radioprotection et de Sûreté Nucléaire (IRSN) DPAM/SEMIC/LIMSI, Cadarache, BP 3, \\ 13115 Saint-Paul-Lez-Durance, France \\ 2 Institut de Mécanique des Fluides de Toulouse (IMFT), 1 allée du Pofesseur Camille Soula, 31400 Toulouse, France
}

Reçu le 29 avril 2011, accepté le 11 mai 2011

\begin{abstract}
Résumé - Nous étudions expérimentalement et numériquement la dynamique de bulles d'air isolées traversant l'interface initialement plane entre deux liquides newtoniens. Les liquides utilisés dans les expériences nous permettent d'obtenir des rapports de viscosité qui peuvent varier de deux ordres de grandeur entre les deux couches et dans une moindre mesure, de faire varier le contraste de densité et la tension interfaciale rencontrés par la bulle lors de la traversée de l'interface. Deux dispositifs d'injection différents sont utilisés afin d'obtenir des bulles dont les diamètres s'échelonnent typiquement entre $1 \mathrm{~mm}$ et $2 \mathrm{~cm}$ et dont les plus grosses adoptent une forme torique. Nous réalisons en parallèle des simulations numériques axisymétriques à l'aide de deux approches distinctes basées pour l'une sur un modèle de capture d'interface (de type VOF mais sans reconstruction d'interface), pour l'autre sur un modèle de Cahn-Hilliard couplé aux équations de Navier-Stokes.
\end{abstract}

Mots clés: Bulles / écoulement triphasique / interface liquide-liquide / simulation numérique directe

\begin{abstract}
Dynamics of bubbles crossing a liquid-liquid interface. We investigate experimentally and computationally the passage of air bubbles through an initially flat horizontal interface separating two Newtonian liquids. We use various liquids in such a way that their viscosity ratio can vary by more than two orders of magnitude; the density contrast and the interfacial tension also vary to a certain extent. Two different injection systems are used and allow us to generate bubbles with diameters ranging typically from $1 \mathrm{~mm}$ to $2 \mathrm{~cm}$, the biggest bubbles being toroidal. In parallel we carry out axisymmetric computations based on two distinct approaches, namely an interface capturing technique (a VOF approach without interface reconstruction) and a Cahn-Hilliard model coupled with the Navier-Stokes equations.
\end{abstract}

Key words: Bubbles / triphasic flow / liquid-liquid interface / direct numerical simulation

\section{Introduction}

Au cours d'un hypothétique accident au sein d'un réacteur à eau pressurisée, la dégradation du cœur peut entraîner l'apparition d'une configuration stratifiée métal/oxyde dans le puits de cuve, traversée par un flux gazeux. Les transferts entre les deux phases métallique et oxyde peuvent accélérer l'ablation du puits de cuve dans une direction (horizontale ou verticale) et la quantification de ces échanges reste un problème ouvert qui pèse sur la fiabilité des simulations actuelles d'accident grave.

Les phénomènes hydrodynamiques mis en jeu lorsque des bulles de gaz, des gouttes ou des particules solides approchent une interface initialement plane et horizontale séparant deux liquides non miscibles puis la traversent éventuellement ont fait l'objet de plusieurs

${ }^{a}$ Auteur pour correspondance : romain.bonhomme@imft.fr travaux tant expérimentaux que numériques. Une analyse très complète du problème de l'approche de l'interface (mais pas de sa traversée) a été réalisée dans le domaine des faibles nombres de Reynolds par Manga et Stone [1] en combinant expériences (avec plusieurs fluides), simulations numériques (par une méthode d'intégrales de frontière) et approche asymptotique. Des expériences à grand nombre de Reynolds dans un canal confiné entre deux plaques ont été menées par Eames et Duursma [2] et Bush et Eames [3] afin d'analyser l'entraînement du liquide inférieur dans le sillage d'une bulle ayant préalablement traversé l'interface. Plus récemment, Kemiha et al. [4] et Dietrich et al. [5] ont étudié à l'aide d'une technique de vélocimétrie par images de particules le passage de bulles d'air au travers de l'interface séparant un liquide newtonien ou rhéofluidifiant d'une couche supérieure constituée d'huile de silicone. 

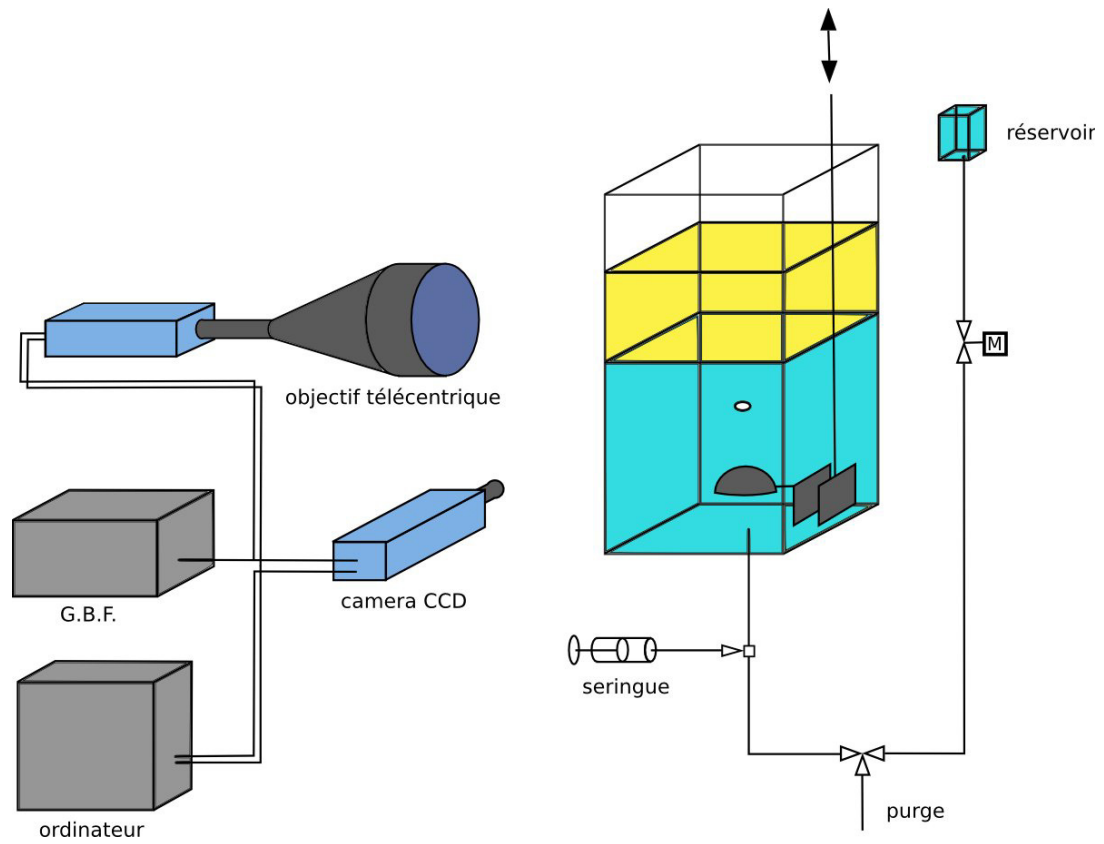

Fig. 1. Dispositif expérimental intégrant le système d'injection avec demi-coque.

En termes de simulation, Shopov et Minev [6] utilisant une technique de maillage adaptatif couplée à une approche en éléments-finis ont pu simuler l'approche d'une interface initialement plane par des bulles et des gouttes pour des nombres de Reynolds inférieurs à la centaine et des nombres de Bond inférieurs à 20. Plus récemment, Cranga et al. [7] et Bonometti [8] ont étendu une approche de type VOF au cas triphasique et ont simulé des traversées d'interface par des bulles dans des géométries axisymétriques mais également tridimensionnelles dans des régimes d'écoulement variés. Boyer et al. [9] ont d'autre part développé un modèle de Cahn-Hilliard couplé aux équations de Navier-Stokes qui leur a permis de simuler la traversée d'une interface liquide-liquide par des bulles.

Notre objectif général est d'approfondir, en combinant les approches expérimentales et numériques aujourd'hui disponibles (notamment les deux dernières approches numériques citées ci-dessus), la compréhension des mécanismes hydrodynamiques mis en jeu dans ce problème, ainsi que d'explorer une gamme de configurations plus large que celle des études antérieures.

\section{Dispositif expérimental}

Les expériences ont été réalisées à température constante $\left(20 \pm 1^{\circ} \mathrm{C}\right)$ dans une colonne en verre de section carrée, de dimensions $20 \times 20 \times 40 \mathrm{~cm}$. Deux faces de la cuve sont composées de verre B270 Superwite ${ }^{\circledR}$ afin de limiter les aberrations optiques lors de l'acquisition d'image. Chacune des parois de la colonne est traitée avec un composé hydrophobe Rain- $\mathrm{X}^{\circledR}$ dans sa partie supérieure dans le but d'obtenir une interface liquide-liquide totalement plane le long de la ligne triple verre/phase aqueuse/phase huileuse. Le socle de la colonne est en PMMA ; il est amovible et intègre plusieurs systèmes d'injection interchangeables.

Deux dispositifs d'injection de gaz ont été mis au point dans cette étude : un système avec demi-coque présenté en figure 1, ainsi qu'un système utilisant un piston. Le premier se compose d'une cuiller de diamètre intérieur $60 \mathrm{~mm}$ qui peut tourner autour d'un axe horizontal. Un volume d'air prédéterminé est tout d'abord injecté sous la cuiller à l'aide d'une seringue. La cuiller est ensuite retournée pour libérer la bulle emprisonnée. Le second système consiste à injecter manuellement et de manière rapide au moyen d'un piston l'air emprisonné dans un cylindre fermé. Les bulles sub-centimétriques prennent des formes variées, sphériques pour les plus petites, en calotte sphérique pour les plus grosses. Les bulles de volume plus grand, obtenues au moyen du deuxième dispositif, prennent préférentiellement une forme torique.

Les liquides lourds utilisés lors des expérimentations sont des mélanges d'eau et de glycérine de concentrations différentes. Les liquides légers sont des huiles de silicone 47V10, 47V100 et 47V500. Les propriétés physiques de ces fluides ont été mesurées à $20 \pm 1{ }^{\circ} \mathrm{C}$ et sont référencées dans le tableau 1.

Afin d'obtenir une visualisation tridimensionnelle de l'écoulement, deux caméras sont positionnées de manière frontale et latérale par rapport à la cuve. Nous avons utilisé des caméras haute cadence PCO1200 HS synchronisées à 350 images par seconde. Les images obtenues ont une résolution de $1024 \times 1280$ pixels et sont encodées sur 16 bits de niveaux de gris. La caméra frontale est connectée à un objectif télécentrique TC 4M 120 dans le but de réduire les distorsions. Le système a été calibré afin que chaque caméra détecte un champ fixe de $108,9 \times 87,2 \mathrm{~mm}^{2}$. 
Tableau 1. Propriétés mesurées des liquides à $20 \pm 1{ }^{\circ} \mathrm{C}$.

\begin{tabular}{lccc}
\hline Liquide & $\begin{array}{c}\text { Masse volumique } \\
\left(\mathrm{kg} . \mathrm{m}^{-3}\right)\end{array}$ & $\begin{array}{c}\text { Viscosité } \\
(\mathrm{MPa} . \mathrm{s})\end{array}$ & $\begin{array}{c}\text { Tension de surface liquide-air } \\
\left(\mathrm{mN.m}^{-1}\right)\end{array}$ \\
\hline Eau & 997 & 1,002 & 69,4 \\
Glycérine 85 \% + eau & 1211 & 102,6 & 48,7 \\
Glycérine 95 \% + eau & 1244 & 550,1 & 45,2 \\
Huile 47V10 & 932 & 9,6 & 20,2 \\
Huile 47V100 & 961 & 113,8 & 20,7 \\
Huile 47V500 & 965 & 530,7 & 21,0 \\
\hline
\end{tabular}

\begin{tabular}{l|ccc}
\hline $\begin{array}{c}\text { Tensions interfaciales } \\
\left(\mathrm{mN}^{-1} \mathrm{~m}^{-1}\right)\end{array}$ & Huile 47V10 & Huile 47V100 & Huile 47V500 \\
\hline Eau & 19,7 & 14,3 & 13,7 \\
Glycérine 85 \% + eau & 28,8 & 30,0 & 29,9 \\
Glycérine 95 \% + eau & 27,8 & 27,1 & 28,0 \\
\hline
\end{tabular}

\section{Observations expérimentales}

Plusieurs comportements contrastés peuvent être observés lors du passage de bulles à travers une interface liquide-liquide.

Dans une configuration où les deux liquides sont très visqueux (viscosités supérieures à $500 \mathrm{MPa}$.s dans la Fig. 2), et pour des bulles de tailles centimétriques, l'écoulement est axisymétrique. Une colonne de liquide lourd est alors entraînée dans le liquide léger à la traversée de l'interface.

Un deuxième comportement peut être observé sur la figure 3 : la bulle est fortement déformée au passage de l'interface à cause du contraste de viscosité entre les deux liquides. L'arrière de la bulle est piégé dans le fluide lourd alors que le front tend à s'échapper dans le liquide supérieur de moindre viscosité. L'étirement ainsi créé entre l'avant et l'arrière de la bulle peut être tel que la bulle se scinde.

Une autre configuration est obtenue lorsqu'une bulle torique traverse une interface eau-huile. L'organisation de l'écoulement autour de la bulle permet la formation d'une enveloppe d'eau qui entoure entièrement la bulle, sans ligne triple. Cette enveloppe suit la bulle lors de son ascension dans la phase supérieure.

\section{Simulations numériques par une approche de capture d'interfaces}

Une approche VOF sans reconstruction d'interface est employée dans le code JADIM de l'Institut de Mécanique des Fluides de Toulouse pour la simulation d'écoulements diphasiques. Nous utilisons une extension du modèle diphasique de Bonometti et Magnaudet [10] sous les hypothèses que les fluides sont newtoniens, que l'écoulement est incompressible et que les tensions interfaciales sont uniformes.

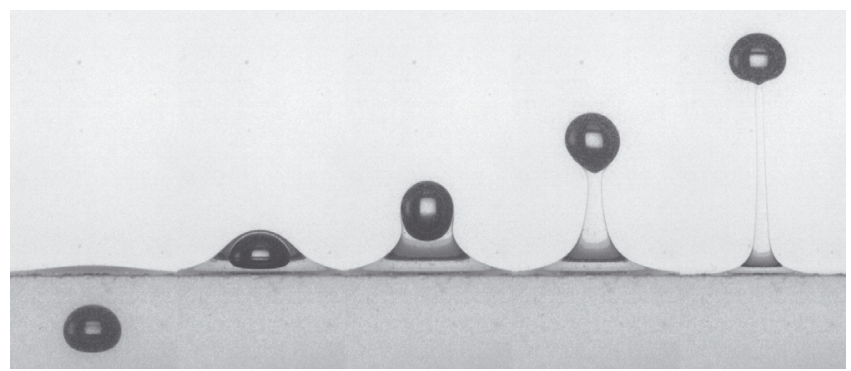

Fig. 2. Passage d'une bulle de $0,38 \mathrm{ml}$ à travers une interface eau-glycérine $95 \%$-huile 47V500. L'intervalle de temps $\Delta t$ entre chaque image est de $0,1429 \mathrm{~s}$. En notant 1 l'indice relatif à la bulle, 2 à la phase lourde, et $V$ le volume de la bulle : le diamètre équivalent de la bulle $d=(6 \mathrm{~V} / \pi)^{\frac{1}{3}}$ est égal à $9 \mathrm{~mm}$, les nombres d'Archimède $A r=\left[\varrho_{2}\left(\varrho_{2}-\varrho_{1}\right) g d^{3}\right]^{\frac{1}{2}} \eta_{2}^{-1}$ et de Bond $B o=\left(\varrho_{2}-\varrho_{1}\right) g d^{2} \sigma_{12}^{-1}$ associés au liquide inférieur valent respectivement 6 et 22 .

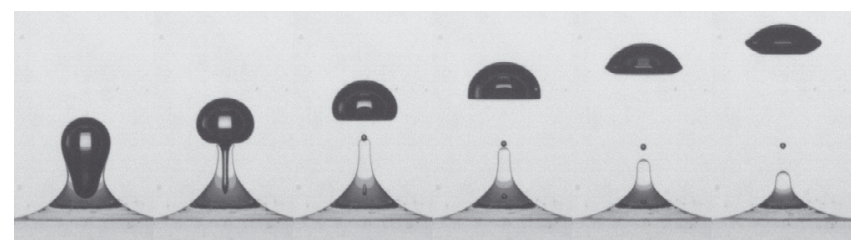

Fig. 3. Passage d'une bulle de $0,18 \mathrm{ml}$ à travers une interface eau-glycérine $95 \%$-huile 47V10. L'intervalle de temps $\Delta t$ entre chaque image est de 0,01429 s. $d=7 \mathrm{~mm}, A r=4, B o=13$.

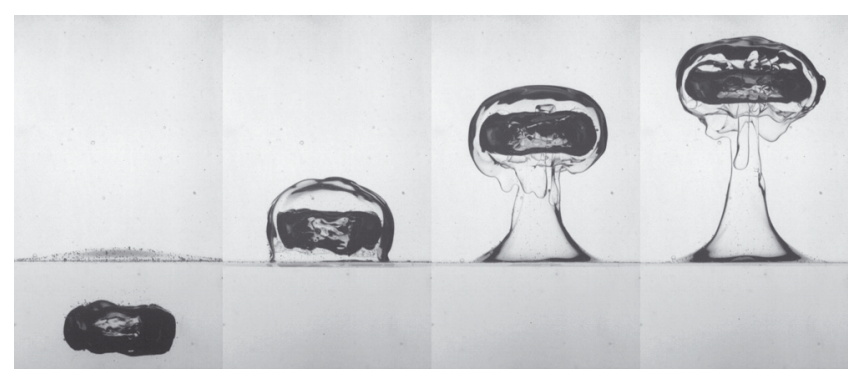

Fig. 4. Passage d'une bulle torique de $3,2 \mathrm{ml}$ à travers une interface eau-huile $47 \mathrm{~V} 10$. L'intervalle de temps $\Delta t$ entre chaque image est de 0,06 s. $d=18 \mathrm{~mm}, A r=7522, B o=46$. 


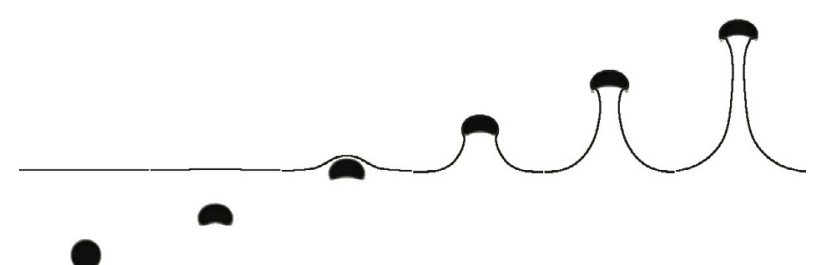

Fig. 5. Simulation numérique VOF obtenue pour les conditions indiquées dans le tableau 2. L'intervalle de temps entre chaque image est de $\Delta t=0,0894 \mathrm{~s} . d=10 \mathrm{~mm}, A r=16$, $B o=31$.

Chacune des phases est repérée par un taux de présence $c_{i}(i=1,2,3)$ compris entre 0 (absence de phase) et 1 (dans la phase), soumis à l'équation de transport

$$
\frac{\partial c_{i}}{\partial t}+\mathbf{u} \cdot \nabla c_{i}=0, \quad \text { pour } i=1,2,3
$$

où $\mathbf{u}$ est la vitesse locale du fluide.

Cette équation est discrétisée en volumes finis et résolue selon un schéma Flux Corrected Transport (FCT) opérant direction par direction.

La masse volumique $\varrho$ et la viscosité $\eta$ du milieu triphasique local sont alors évaluées par interpolation linéaire à partir des taux de présence $c_{i}$

$$
\varrho=\sum_{i} c_{i} \varrho_{i} \quad ; \quad \eta=\sum_{i} c_{i} \eta_{i}
$$

Les forces capillaires sont transformées en forces volumiques selon le modèle Continuum Surface Force de Brackbill et al. [11], étendu au cas triphasique par Cranga et al. [7]. En notant $p$ la pression du milieu triphasique et $\mathbf{g}$ la gravité, les équations de Navier-Stokes incompressibles du modèle s'écrivent

$$
\left\{\begin{aligned}
\frac{\partial \mathbf{u}}{\partial t}+\mathbf{u} \cdot \nabla \mathbf{u}= & -\frac{1}{\varrho} \nabla p+\frac{1}{\varrho} \nabla \cdot\left[\eta\left(\nabla \mathbf{u}+\nabla \mathbf{u}^{\mathrm{T}}\right)\right] \\
& +\mathbf{g}-\sum_{i, j} \delta_{i j} \frac{\left(c_{i}+c_{j}\right)^{2} \sigma_{i j}}{\varrho_{i}+\varrho_{j}} \nabla \cdot\left[\frac{\nabla c_{i j}}{\mid \nabla c_{i j}}\right] \nabla c_{i j} \\
\nabla \cdot \mathbf{u}=0 &
\end{aligned}\right.
$$

avec $c_{i j}=\frac{c_{i}}{c_{i}+c_{j}}$ et $\delta_{i j}=\left\{\begin{array}{l}1 \text { si }\left|\nabla c_{i}\right|\left|\nabla c_{j}\right| \neq 0 \\ 0 \text { sinon }\end{array}\right.$.

L'avancement en temps des équations de NavierStokes est réalisé par un schéma hybride de Runge Kutta/Crank-Nicolson. La vitesse est ensuite rendue à divergence nulle par une méthode de projection.

Un résultat de simulation numérique obtenu par cette approche est présenté en figure 5. On note l'entraînement de liquide lourd dans la phase supérieure. Les propriétés physiques de l'écoulement sont référencées dans le tableau 2.

\section{Simulations numériques par un modèle Cahn-Hilliard/Navier-Stokes}

Une deuxième approche a été développée à l'Institut de Radioprotection et de Sûreté Nucléaire à Cadarache

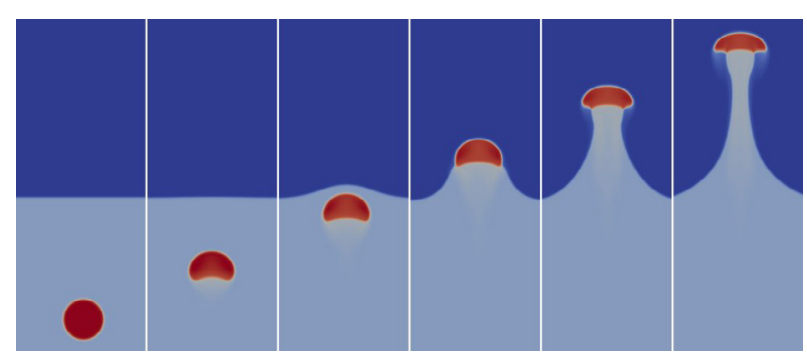

Fig. 6. Simulation Cahn-Hilliard/Navier-Stokes de la situation physique décrite par la figure 4 .

à partir de la plateforme de calculs PELICANS. Elle consiste à coupler les équations de Cahn-Hilliard étendues au cas triphasique par Boyer et al. [9], aux équations de Navier-Stokes incompressibles.

L'épaisseur des interfaces est considérée non-nulle et de valeur $\epsilon$. Chacune des phases est repérée par un taux de présence $c_{i}(i=1,2,3)$ compris entre 0 (absence de phase) et 1 (dans la phase), soumis aux équations de Cahn-Hilliard

$\left\{\begin{array}{cc}\frac{\partial c_{i}}{\partial t}+\mathbf{u} \cdot \nabla c_{i}=\nabla \cdot\left(\frac{M}{\Sigma_{i}} \nabla \mu_{i}\right) & \text { pour } i=1,2,3 \\ \mu_{i}=-\frac{3}{4} \Sigma_{i} \epsilon \nabla^{2} c_{i}+\frac{4 \Sigma_{T}}{\epsilon} \sum_{j \neq i}\left(\frac{1}{\Sigma_{i}}\left(\frac{\partial F}{\partial c_{i}}-\frac{\partial F}{\partial c_{j}}\right)\right) & \text { pour } i=1,2,3\end{array}\right.$

où $M$ est la mobilité et $F$ le potentiel algébriquement consistant de Cahn-Hilliard triphasique défini par Boyer et al. [12] selon la formule

$$
F=\sum_{i} \frac{\Sigma_{i}}{2} c_{i}^{2}\left(1-c_{i}\right)^{2}+3 \Lambda c_{1}^{2} c_{2}^{2} c_{3}^{2}
$$

où la fonction $\Lambda$ est positive, $\Sigma_{i}=\sigma_{i j}+\sigma_{i k}-\sigma_{j k}$ avec $i, j, k \in\{1,2,3\}, i \neq j \neq k$, et où $\frac{3}{\Sigma_{T}}=\sum_{i} \frac{1}{\Sigma_{i}}$.

Ces équations de taux de présence sont discrétisées en espace par une méthode de Galerkin en éléments-finis avec raffinement local adaptatif, et selon un schéma semiimplicite en temps.

La masse volumique $\varrho$ et la viscosité $\eta$ du mélange sont alors évaluées comme dans (2). Les équations de NavierStokes associées à cette approche peuvent s'écrire sous la forme

$$
\left\{\begin{array}{l}
\sqrt{\varrho} \frac{\partial}{\partial t}(\sqrt{\varrho} \mathbf{u})+(\varrho \mathbf{u} \cdot \nabla) \mathbf{u}+\frac{1}{2} \mathbf{u} \nabla \cdot(\varrho \mathbf{u}) \\
-\nabla \cdot\left[\eta\left(\nabla \mathbf{u}+\nabla \mathbf{u}^{\mathrm{T}}\right)\right]+\nabla p=\varrho \mathbf{g}+\sum_{i} \mu_{i} \nabla c_{i} \\
\nabla \cdot \mathbf{u}=0
\end{array}\right.
$$

Les équations de Navier-Stokes sont discrétisées en temps par une méthode de projection incrémentale.

Un résultat de simulation numérique obtenu à partir de ce modèle est présenté en figure 6 . Il est à mettre en relation avec le résultat de la simulation par méthode VOF présenté sur la figure 5 puisque les propriétés physiques des fluides sont identiques. La mobilité $M$ est prise égale à $1,42 \times 10^{-8}$, et l'épaisseur d'interface $\epsilon$ à 0,001 . 
Tableau 2. Propriétés et indexation des fluides utilisées lors des simulations numériques.

\begin{tabular}{l|ccc|ccc}
\hline Volume de la bulle $(\mathrm{ml})$ & \multicolumn{3}{|c|}{$V$} & & 0.52 & \\
Masses volumiques $\left(\mathrm{kg} . \mathrm{m}^{-3}\right)$ & $\varrho_{1}$ & $\varrho_{2}$ & $\varrho_{3}$ & 9,31 & 1000 & 931 \\
Viscosités dynamiques $(\mathrm{mPa} . \mathrm{s})$ & $\eta_{1}$ & $\eta_{2}$ & $\eta_{3}$ & 2,01 & 201 & 94,7 \\
Tensions de surface $\left(\mathrm{mN} \cdot \mathrm{m}^{-1}\right)$ & $\sigma_{12}$ & $\sigma_{13}$ & & 31,0 & 16,5 & \\
Tension interfaciale $\left(\mathrm{mN} . \mathrm{m}^{-1}\right)$ & $\sigma_{23}$ & & & 12,7 & & \\
\hline
\end{tabular}

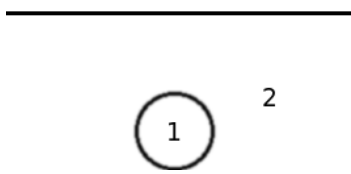

\section{Conclusions}

Nous avons observé plusieurs comportements contrastés lorsque des bulles traversent l'interface séparant deux liquides non miscibles initialement au repos. Les expériences mettent en lumière les déformations significatives des interfaces liquide-liquide et liquide-gaz au cours de l'approche et de la traversée de la bulle, ainsi que de grandes différences dans la forme et le volume de la colonne de fluide lourd entraîné.

Des simulations numériques directes ont été réalisées à l'aide de deux codes de calcul utilisant respectivement des modèles VOF sans reconstruction d'interface et CahnHilliard/Navier-Stokes. Une bonne similarité entre les résultats de ces simulations est à noter pour des liquides à fortes viscosités.

Notre objectif est maintenant de mener des comparaisons plus poussées avec les résultats expérimentaux afin notamment d'identifier les éventuelles limitations de l'approche numérique.

Remerciements. Nous remercions G. Ehses pour l'élaboration du dispositif expérimental, ainsi que S. Cazin pour sa contribution à la métrologie et au traitement des images.

\section{Références}

[1] M. Manga, H.A. Stone, Low Reynolds number motion of bubbles, drops and rigid spheres through fluid-fluid interfaces, J. Fluid Mech. 287 (1995) 279-298

[2] I. Eames, G. Duursma, Displacement of horizontal layers by bubbles injected into fluidised beds, Chem. Eng. Sci. 52 (1997) 2697-2705
[3] J.W.M. Bush, I. Eames, Fluid displacement by high Reynolds number bubble motion in a thin gap, Int. J. Multiphase Flow 24 (1998) 411-430

[4] M. Kemiha, E. Olmos, W. Fei, S. Poncin, H.Z. Li, Passage of a gas bubble through a liquid-liquid interface, Ind. Eng. Chem. Res. 46 (2007) 6099-6104

[5] N. Dietrich, S. Poncin, S. Pheulpin, H.Z. Li, Passage of a bubble through a liquid-liquid interface, AIChE J. 54 (2008) 594-600

[6] P.J. Shopov, P.D. Minev, The unsteady motion of a bubble or drop towards a liquid-liquid interface, J. Fluid Mech. 235 (1992) 123-141

[7] J. Cranga, P. Gardin, D. Huin, J. Magnaudet, Influence of surface pressure and slag layer on bubble bursting in degasser systems, Computational Modeling of Materials, Minerals, and Metals Processing, 2001, pp. 105-114

[8] T. Bonometti, Développement d'une méthode de simulation d'écoulements à bulles et à gouttes, Thèse de doctorat, Institut National Polytechnique de Toulouse, 2005

[9] F. Boyer, C. Lapuerta, S. Minjeaud, B. Piar, M. Quintard, Cahn-Hilliard/Navier-Stokes model for the simulation of three-phase flows, Transp. Porous Media 82 (2010) 463-483

[10] T. Bonometti, J. Magnaudet, An interface-capturing method for incompressible two-phase flows, Validation and application to bubble dynamics, Int. J. Multiphase Flow 33 (2007) 109-133

[11] J.U. Brackbill, D.B. Kothe, C. Zemach, A continuum method for modeling surface tension, J. Comput. Phys. 100 (1992) 335-354

[12] F. Boyer, C. Lapuerta, Study of a three component CahnHilliard flow model, ESAIM: M2AN, 40 (2006) 653-687 